\title{
Recurrent nontuberculous mycobacterial endophthalmitis: a diagnostic conundrum
}

\author{
This article was published in the following Dove Press journal: \\ Clinical Ophthalmology \\ 2 May 2014 \\ Number of times this article has been viewed
}

\author{
Nandini Venkateswaran' \\ Gabrielle Yeaney ${ }^{2}$ \\ Mina Chung ${ }^{3,4}$ \\ Holly B Hindman ${ }^{3,4}$ \\ 'University of Rochester School \\ of Medicine and Dentistry, University \\ of Rochester, ${ }^{2}$ Department of \\ Pathology and Laboratory Medicine, \\ ${ }^{3}$ Flaum Eye Institute, ${ }^{4}$ Center \\ for Visual Science, University of \\ Rochester School of Medicine \\ and Dentistry, Rochester, NY, USA
}

Correspondence: Holly B Hindman Flaum Eye Institute, 60I Elmwood Ave, Box 659, Rochester, NY, USA I 4642

Tel +I 5852765482

Fax +I 5852760292

Email holly_hindman@urmc.rochester.edu
Objective: To report a case of recurrent nontuberculous mycobacterial endophthalmitis in the context of neurotrophic keratopathy secondary to herpes zoster ophthalmicus that had an atypical presentation and complex course, and highlights the challenges of causative organism identification and therapeutic interventions in this condition.

Methods: A retrospective chart review was conducted to determine the visual outcomes of the patient.

Results: A 68-year-old pseudophakic male with long-standing neurotrophic keratopathy and perforated descemetocele managed with cyanoacrylate glue and a contact bandage lens in the left eye, began experiencing recurrent episodes of endophthalmitis after undergoing a penetrating keratoplasty. Several therapeutic procedures including an anterior chamber washout, two pars plana vitrectomies, explantation of the posterior chamber intraocular lens and capsular bag, and multiple intravitreal antimicrobial injections, were performed to which he has ultimately responded favorably, with no signs of infection to date and stable visual acuity. The causative organism of his recurrent infections was initially identified as Mycobacterium abscessus through biochemical testing and 165 ribosomal ribonucleic acid gene sequencing; however, repeat polymerase chain reaction (PCR) and sequencing of the $65 \mathrm{kDa}$ heat shock protein (hsp65) gene for experimental purposes confirmed the accurate identification of the organism to be Mycobacterium chelonae. Given the greater reliability of PCR and sequencing of the hsp65 gene over traditional biochemical tests and culture techniques, $M$. chelonae was likely the infectious agent all along, and the organism was originally misidentified on the basis of less accurate tests.

Conclusion: Recurrent atypical mycobacterial endophthalmitis requires expedient identification and management to prevent poor visual outcomes. Standard biochemical testing can identify the causative organism but is limited by the inability to distinguish between nontuberculous species reliably. We recommend the use of PCR in conjunction with sequencing of the hsp65 gene for reliable differentiation of $M$. chelonae and M. abscessus in atypical mycobacterial ocular infections. Minimum inhibitory concentration antibiotic susceptibility tests on cultured strains are the best guide to antibiotic selection, given the rapidly rising resistance to antimicrobials in atypical mycobacterial species.

Keywords: atypical mycobacteria, herpes zoster ophthalmicus, hsp65, Mycobacterium chelonae, neurotrophic keratopathy, visual outcome

\section{Introduction}

Rapidly-growing nontuberculous mycobacteria (RGNTM) include bacteria such as Mycobacterium chelonae, Mycobacterium abscessus, and Mycobacterium fortuitum. . $^{12}$ These atypical mycobacteria can be acquired from the natural environment and nosocomially. The mycobacteria can infect the eye, resulting in keratitis, conjunctivitis, and endophthalmitis among other ocular infections. ${ }^{1}$ Risk factors for ocular RGNTM infections include trauma, previous corneal infection or surgery, corticosteroid use, and 
systemic immunosuppression. ${ }^{3}$ We report a case of recurrent atypical mycobacterial endophthalmitis in the context of neurotrophic keratopathy secondary to herpes zoster ophthalmicus (HZO) that had an atypical presentation and complex course, and highlight the challenges of causative organism identification and therapeutic interventions in this condition.

\section{Case report}

A 68-year-old pseudophakic male with long-standing neurotrophic keratopathy secondary to $\mathrm{HZO}$ characterized by a non-healing corneal ulcer (Figure 1A), was referred with a recurrent Seidel-positive cornea of the left eye. Medical history was notable for chronic lymphocytic leukemia that was in remission after chemotherapy treatment (oral chlorambucil and combination fludarabine and cyclophosphamide). He developed herpes zoster with ocular involvement soon after completing the chemotherapy. Prior management of the neurotrophic cornea included topical lubricating; antibiotic and steroid drops; oral antivirals; injections of botulinum toxin for induction of ptosis; and amniotic membrane grafts (AMG). A corneal descemetocele had perforated 6 months prior and was managed with cyanoacrylate glue and a bandage contact lens.

Examination revealed cyanoacrylate glue on the central cornea with slow aqueous leakage, diffuse deep vascularization, an inferior stromal infiltrate, and a $1.5 \mathrm{~mm}$ layered hypopyon (Figure 1B). The glue was removed and re-applied after obtaining corneal scrapings, and topical vancomycin and tobramycin were administered empirically. Initial aerobic cultures were positive for micrococcus species, and acid-fast bacilli (AFB) stain was 3+ positive. Subsequent cultures grew M. abscessus. Topical amikacin and moxifloxacin and oral clarithromycin were started. Six weeks later, examination results remained unchanged.
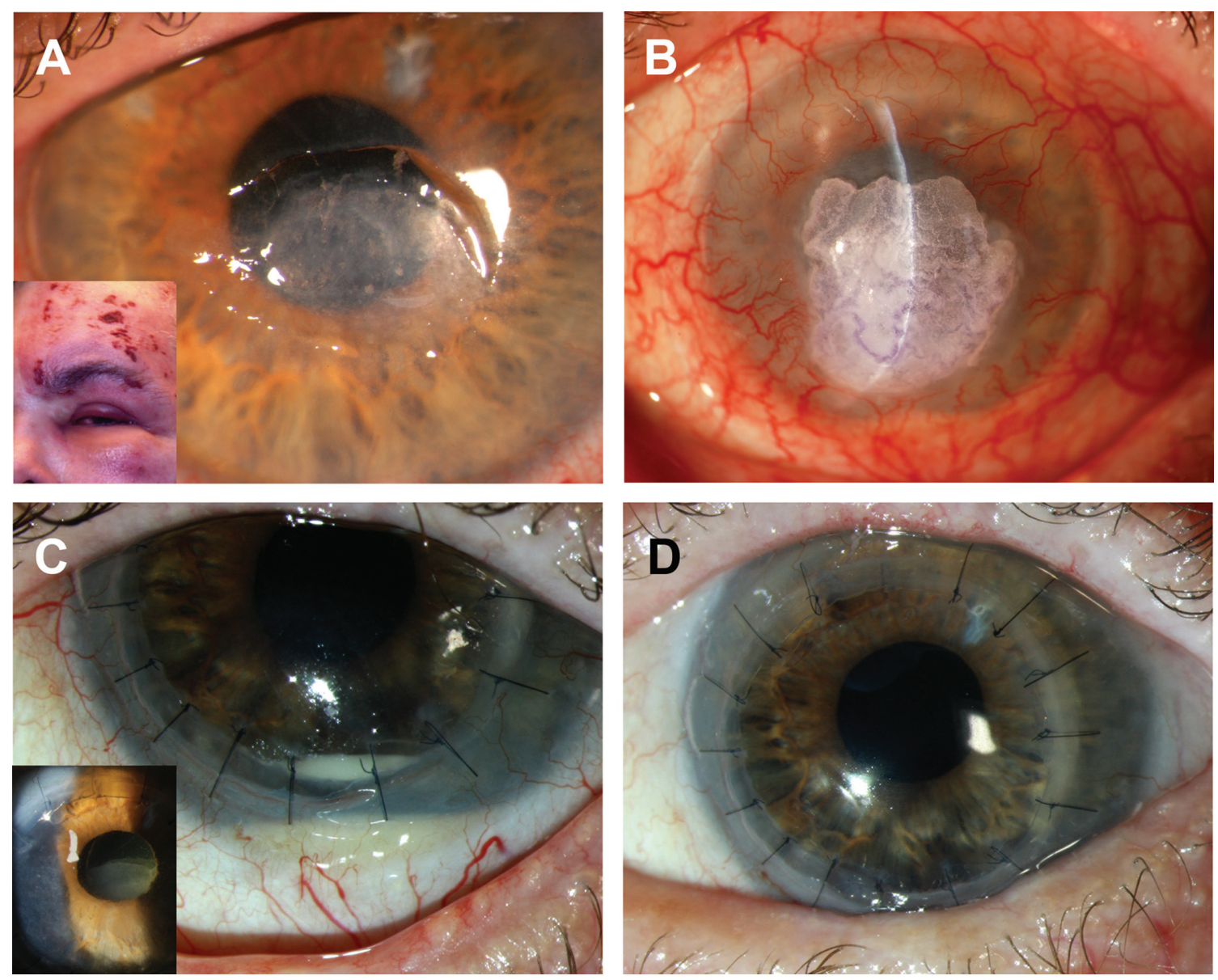

Figure I Clinical photographs.

Notes: (A) Patient's clinical presentation of herpes zoster ophthalmicus in 2002 (inset) with a slit-lamp photograph of his neurotrophic corneal ulcer in 2007. (B) Cyanoacrylate glue had been applied for the management of a perforated corneal descemetocele in April 20I I. (C) Suspected vitreous prolapse from previous cataract surgery or accumulated fibrin that may have allowed passage of mycobacterial past the lens implant into the vitreous cavity (inset), and recurrent hypopyon. (D) Clear corneal graft and anterior chamber I month after the pars plana vitrectomy, anterior chamber washout, and intravitreal injections of antimicrobial and fungal agents (amikacin, $400 \mu \mathrm{g} / 0.1 \mathrm{~mL}$; vancomycin, I mg/0.I mL; and amphotericin B, $5 \mu \mathrm{g} / 0.1 \mathrm{~mL}$ ). 
A therapeutic penetrating keratoplasty $(\mathrm{PK})$ with anterior chamber (AC) washout, AMG, subconjunctival bevacizumab injection, and permanent tarsorrhaphy were performed. AMG was used for its anti-inflammatory, anti-scarring, and wound healing properties on the ocular surface, ${ }^{4}$ and subconjunctival bevacizumab was used to help decrease corneal neovascularization. ${ }^{5}$ Initially, the patient recovered well but returned 20 days post-PK with painless $3+$ white blood cells in the AC. B-scan ultrasonography showed dense retrolental opacity, concerning for endophthalmitis (Figure 2A). Vitreous tap and intravitreal injection of ceftazidime $(2 \mathrm{mg} / 0.1$ $\mathrm{mL})$ and vancomycin $(1 \mathrm{mg} / 0.1 \mathrm{~mL})$ were performed with rapid improvement. Stains and cultures from the tap were negative. Histopathology from the host corneal button subsequently demonstrated AFB, and cultures were reported as M. abscessus (Figure 2B). The eye responded immediately to the administered antibiotics.

However, 9 months post-PK, the patient returned with minimal discomfort. There was no corneal infiltrate, but 4+ white blood cells in the AC, recurrent layered hypopyon (Figure 1C), mild anterior vitritis, and unaffected posterior vitreous were observed. A second vitreous tap and intravitreal injections of ceftazidime $(2 \mathrm{mg} / 0.1 \mathrm{~mL})$ and vancomycin $(1 \mathrm{mg} / 0.1 \mathrm{~mL})$ were administered based on prior responsiveness; however, this time, the patient did not improve. The tap revealed no organisms or cells, and cultures (aerobic, anaerobic, AFB, and fungal) and viral polymerase chain reaction (PCR) (to test for herpes simplex virus 1 and 2, and varicella zoster virus) were negative. Anterior and intermediate uveitis work-ups were performed (to test for tuberculosis, sarcoidosis, human leukocyte antigen B27, Lyme disease, and syphilis), and were negative.

Based on the recent negative work-up and failure to respond to therapy, as well as prior culture results, vitreous and AC biopsies, pars plana vitrectomy (PPV), AC washout, and injection of intravitreal antimicrobial and fungal agents (amikacin, $400 \mu \mathrm{g} / 0.1 \mathrm{~mL}$; vancomycin, $1 \mathrm{mg} / 0.1 \mathrm{~mL}$; and amphotericin B, $5 \mu \mathrm{g} / 0.1 \mathrm{~mL}$ ), were performed. Antibiotic susceptibility testing was performed prior to this procedure on $M$. abscessus isolates grown from the prior host corneal button cultures, and showed high sensitivity to amikacin. Internal stains and cultures of the vitreous sample showed $2+$ AFB positive staining, and culture results were reported as $M$. abscessus complex. Vitreous samples were also sent out to a validated laboratory (the University of Washington's, clinical microbiology laboratory; Seattle, WA, USA) for PCR and gene sequencing. PCR using primers targeting the $65 \mathrm{kDa}$ heat shock protein (hsp65) gene and the beta subunit of ribonucleic acid polymerase (rpoB) gene was negative. However, sequencing of the $16 \mathrm{~S}$ ribosomal ribonucleic acid $(r R N A)$ gene was positive for M. abscessus/chelonae complex. Based upon the biochemical testing and gene sequencing results, the presumed cause of infection was M. abscessus.

After surgery, the eye again returned to a quiet state without evidence of infection or inflammation (Figure 1D), until the patient returned 3 months post-vitrectomy with painless clouding of vision in the left eye. Examination revealed presence of a layered hypopyon in the $\mathrm{AC}$ and 2+ inflammatory cells in the vitreous. Concern for recurrence of M. abscessus endophthalmitis prompted intravitreal injection of amikacin
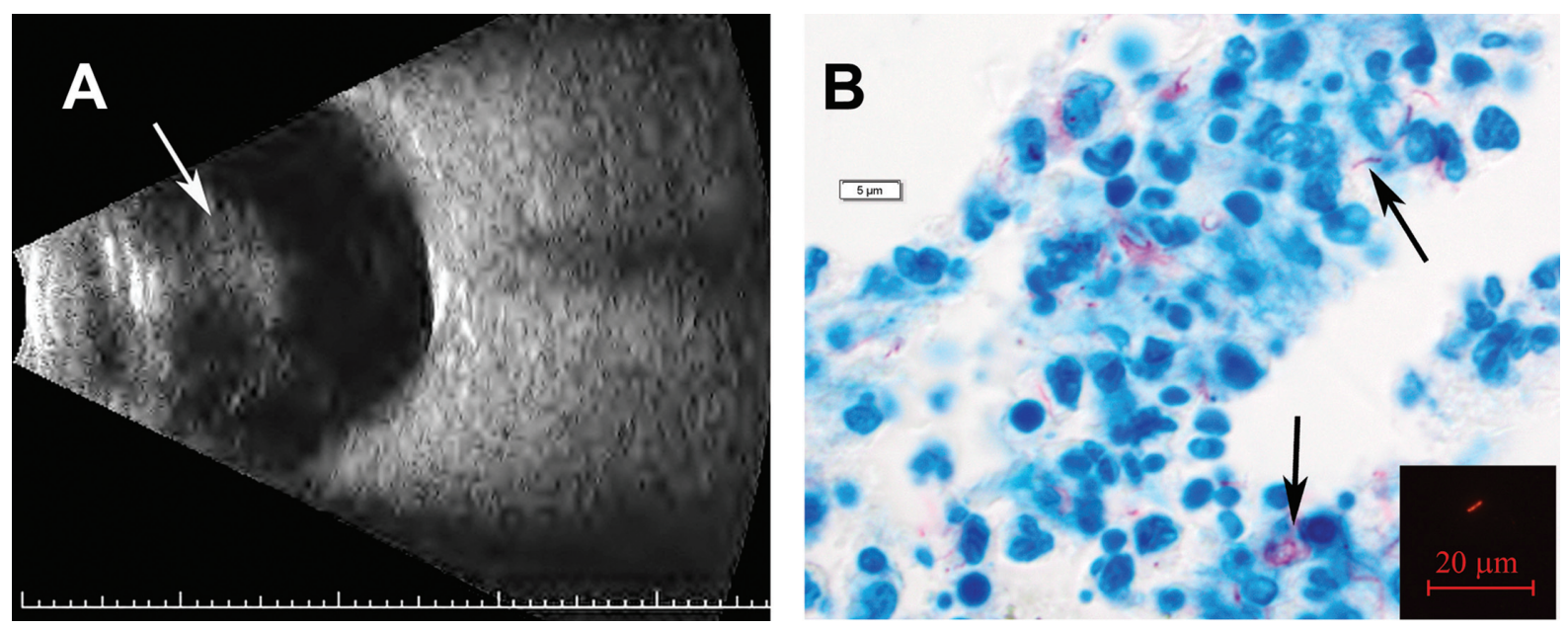

Figure 2 Diagnostic images.

Notes: (A) B-scan ocular ultrasound demonstrating vitreous opacification (arrow) 20 days after the therapeutic penetrating keratoplasty. (B) Ziehl-Neelsen staining of the corneal host button showing red-stained acid-fast bacilli (arrows). Inset shows $2+$ acid-fast rhodamine stain with potassium permanganate counterstain capturing one bacillus per high-powered field from the vitreous cassette obtained by pars plana vitrectomy. 
(400 $\mu \mathrm{g} / 0.1 \mathrm{~mL})$, but activity persisted despite an additional injection of amikacin $(400 \mu \mathrm{g} / 0.1 \mathrm{~mL})$ the following week. PPV and explantation of the posterior chamber intraocular lens and capsular bag with intravitreal injection of amikacin (400 $\mu \mathrm{g} / 0.1 \mathrm{~mL})$, were then performed to eradicate mycobacteria that could be sequestered within the capsular bag. Pathologic examination of the capsular bag showed rare neutrophils, and Brown-Brenn and AFB stains were negative for bacteria and acid-fast organisms. However, AFB cultures of the vitreous cassette from the second vitrectomy were again reported as positive for $M$. abscessus, further substantiating the presumptive diagnosis of recurrent $M$. abscessus endophthalmitis. Since then, the eye has remained quiet without signs of infection for 11 months, and visual acuity is 20/70 with contact lens correction. The patient continues to be monitored closely by his ophthalmologists for recurrence.

Given the challenges encountered in identifying this organism, repeat PCR and gene sequencing were performed at the University of Rochester's clinical microbiology laboratory on culture growths from previously obtained vitreous samples from the two PPV procedures, for experimental purposes. 16S rRNA gene sequencing was first performed, which identified M. abscessus/chelonae complex. PCR as well as sequencing of the hsp65 gene was subsequently performed to differentiate between the two organisms. The result was the identification of $M$. chelonae. Since all prior biochemical testing had identified the organism as $M$. abscessus, and the initial send-out sequencing of the $16 \mathrm{~S}$ $r R N A$ gene had identified $M$. abscessus/chelonae complex, M. abscessus had been considered the most likely causative organism throughout the patient's clinical course. However, this discrepancy in identification upon repeat PCR and gene sequencing of the $h s p 65$ gene was surprising. It is unlikely that both organisms co-existed in the patient's eye during his episodes of recurrent endophthalmitis. Given the greater reliability of PCR and sequencing of the hsp65 gene over traditional biochemical tests and culture techniques, ${ }^{6-8}$ we now believe that $M$. chelonae was likely the infectious agent all along and that the organism was initially misidentified on the basis of less accurate tests.

\section{Discussion}

M. chelonae endophthalmitis is a grave condition associated with poor visual outcomes. ${ }^{9-15}$ Causes of $M$. chelonae endophthalmitis include prior cataract surgery, ${ }^{9-12}$ glaucoma surgery, ${ }^{13}$ iridocyclitis, ${ }^{14}$ intravitreal steroid injections, ${ }^{9,15}$ neurotrophic cornea, ${ }^{9}$ and systemic immunosuppression. ${ }^{16,17}$ The patient had several risk factors for an ocular RGNTM infection: 1) an immunosuppressed state after completion of chemotherapy during which herpes zoster infections are more common; 2) a neurotrophic cornea secondary to HZO with a non-healing ulcer and recurrent perforations; and 3) multiple diagnostic and therapeutic ocular surgeries, long-term antibiotic therapy, and steroid immunosuppression during periods of active infection. The mycobacteria likely gained entry via the perforated descemetocele. The long-standing cyanoacrylate glue and bandage contact lens used to manage the open globe may have created the milieu for the mycobacteria to thrive. Given the neurotrophic cornea and the difficulty in visualizing corneal changes, a smoldering mycobacterial keratitis may have been long-standing but difficult to detect and monitor. Absence of the crystalline lens may have allowed passage of the organism into the vitreous during the therapeutic PK.

The cause of the early endophthalmitis is unclear, as the vitreous tap was negative for bacterial, viral, and fungal agents. Although mycobacteria may have been implicated, the eye cleared in response to vancomycin and ceftazidime, antibiotics to which $M$. chelonae has a low response due to its relatively impermeable lipid cell envelope, drug export systems, biofilm development, and intrinsic B-lactamase activity which would inactivate ceftazidime. ${ }^{18}$ It is possible that the patient had a polymicrobial infection. Nonetheless, despite complete resolution of initial activity, endophthalmitis recurred 9 months later, this time clearly caused by RGNTM. M. chelonae may have been sequestered in the eye for the duration, initially kept indolent by low susceptibility to the vancomycin, ceftazidime, and poorly penetrant topical amikacin, and then reactivated as these medications were discontinued. Additionally, as the topical steroids were tapered, the local inflammatory reaction may have increased, resulting in the return of clinical signs of the latent infection.

Although the PPV, AC washout, and intravitreal injection of antimicrobials were initially effective, endophthalmitis recurred and was unresponsive to additional intravitreal amikacin injections, perhaps due to development of microbial resistance. Explantation of the posterior chamber intraocular lens and capsular bag was performed to clear a potential nidus of residual mycobacterial infection. Yet, the pathology results did not identify any mycobacteria within the intraocular lens/capsular bag complex. Alternative foci of sequestration of the mycobacteria could have been in areas posterior to the iris or near the ciliary body.

The patient's presentation and post-operative course were atypical. Patients with RGNTM endophthalmitis typically present with pain, decreased vision, and irritation. ${ }^{3,9,19}$ Nerve injury from the underlying $\mathrm{HZO}$ and the previous PK 
may have altered this classic presentation. ${ }^{20}$ Post-operative recovery of $M$. chelonae endophthalmitis often results in markedly diminished vision ${ }^{9-11,13,15}$ and enucleation ${ }^{9,12,14}$ due to delayed diagnosis and the devastating nature of the infection. Fortunately, the patient was able to achieve a highly favorable visual outcome despite his protracted clinical course.

Correct identification of the causative organism throughout the patient's clinical course proved to be an unforeseen challenge. M. abscessus, while initially categorized as a subspecies of $M$. chelonae, was determined to be a separate species in 1992 after DNA hybridization studies. ${ }^{2}$ Given the many biochemical similarities between $M$. abscessus and M. chelonae, clinical laboratories often fail to distinguish between these organisms at the species level with standard biochemical testing. ${ }^{6-8}$ Two biochemical tests that are often useful for identifying these organisms at the species level are 5\% sodium chloride tolerance and utilization of sodium citrate. ${ }^{6,7}$ M. abscessus has been shown to grow on Lowenstein-Jensen (LJ) medium with 5\% sodium chloride but fails to grow on LJ medium with sodium citrate, while $M$. chelonae tends to grow in LJ medium with sodium citrate but is unable to grow on LJ medium with $5 \%$ sodium chloride. ${ }^{6}$ However, these tests require up to 4 weeks to complete, ${ }^{6}$ which can delay proper identification and introduce opportunity for error. In this case, the results from these phenotypic tests had guided the initial identification of M. abscessus from cultures, but the discrepancy in the species identification was likely due to inherent inaccuracies in these tests.

Molecular methods are the most reliable means for the identification of RGNTM. ${ }^{6-8}$ PCR is a rapid, specific, and cost-effective means of identification of RGNTM to the species level that can be performed in parallel with standard biochemical tests; $;^{2,6,7}$ however, it often requires outsourcing to validated laboratories, requiring additional time and resources. Gene sequencing also serves as an effective option for identification of RGNTM. ${ }^{6}$ Genetic loci typically used for mycobacteria species identification with PCR or gene sequencing include the $16 \mathrm{~S} r R N A$ gene, the $16 \mathrm{~S}-23 \mathrm{~S}$ spacer region, the $r p o B$ gene, and the $h s p 65$ gene. ${ }^{7}$ Sequencing of the $16 S$ rRNA gene is insufficient to differentiate $M$. abscessus and $M$. chelonae due to high sequence conservation throughout this gene between the two species, with the sequence being identical in the proximal 500 bases of the gene that are sequenced for species identification. ${ }^{7}$ PCR with primers targeted toward the $h s p 65$ gene followed by direct sequencing of this gene is the most reliable method of differentiation, given the high sequence variation in the $h s p 65$ gene between M. abscessus and M. chelonae as well as other RGNTM. ${ }^{6,8}$
In this case, PCR plus an important second step, gene sequencing of the $h s p 65$ gene, helped clinch the identification of the causative organism. The send-out PCR of the rpoB and the $h s p 65$ genes from the vitreous sample from the initial PPV was negative, perhaps due to the limited concentration of the organism in the diluted vitreous sample. Additional sequencing of the $h s p 65$ gene was not attempted given the negative PCR results. Sequencing of the $16 S$ rRNA gene identified the organism as $M$. abscessus/chelonae complex but did not differentiate between the two. Repeat testing performed on culture growths from the same samples likely had increased concentrations of the organisms that allowed for positive $h s p 65$ gene PCR, as well as sequencing results for M. chelonae. While culture growths have been widely used to identify organisms with biochemical testing, and to guide antibiotic selection, their use for PCR and gene sequencing may facilitate accurate identification of bacterial species and warrants further exploration.

While initial identification of the organism was incorrect, all therapy was primarily guided by clinical observation and antibiotic susceptibilities of the cultured organisms. A study by Brown-Elliot et al found that the most active antimicrobials for M. abscessus were amikacin and clarithromycin/ azithromycin, and for M. chelonae were clarithromycin/ azithromycin, amikacin, tobramycin, and quinolones. ${ }^{1}$ Our patient was treated with topical and intravitreal amikacin based on antibiotic susceptibility testing, and this antimicrobial is an appropriate choice for either M. abscessus or M. chelonae. Although now equipped with the knowledge of the correct identification of the causative organism, clinical management in retrospect would not have been changed.

RGNTM endophthalmitis should be highly considered in the differential diagnosis of refractory AC and vitreous inflammation, as expedient identification and management is necessary to prevent poor visual outcomes. Standard biochemical testing can identify the causative organism but is limited by the inability to distinguish between RGNTM species reliably, and should not be used as the sole means of organism identification. We recommend the use of PCR in conjunction with sequencing of the $h s p 65$ gene for reliable differentiation of $M$. chelonae and M. abscessus in RGNTM ocular infections. ${ }^{6-8}$ While PCR and gene sequencing have conventionally been performed directly on patient samples, performing such testing on culture growths can be considered to increase the bacterial load and facilitate the identification of the organism. Cultures remain critical to therapy, as minimum inhibitory concentration antibiotic susceptibility tests on cultured strains are the best guide to antibiotic selection, 
given the rapidly rising resistance to antimicrobials within RGNTM strains. ${ }^{18}$

\section{Acknowledgments}

The authors wish to thank the Flaum Eye Institute's Diagnostic Imaging Service for obtaining the clinical images, Adam Weis for obtaining diagnostic images with fluorescence microscopy, and Susan Raub for assisting us with PCR and gene sequencing of our mycobacterial isolates. The authors also wish to acknowledge the financial support for this case report provided by the National Institutes of Health/NEI K23 EY019353 and an unrestricted grant to the University of Rochester's Department of Ophthalmology from the Research to Prevent Blindness Foundation (RPB).

\section{Disclosure}

The authors have no conflicts of interest in this work.

\section{References}

1. Brown-Elliot BA, Mann LB, Hail D, Whitney C, Wallace RJ Jr. Antimicrobial susceptibility of nontuberculous mycobacteria from eye infections. Cornea. 2012;31(8):900-906.

2. Palani D, Kulandai LT, Naraharirao MH, Guruswami S, Ramendra B. Application of polymerase chain reaction-based restriction fragment length polymorphism in typing ocular rapid-growing nontuberculous mycobacterial isolates from three patients with postoperative endophthalmitis. Cornea. 2007;26(6):729-735.

3. Moorthy RS, Valluri S, Rao NA. Nontuberculous mycobacterial ocular and adnexal infections. Surv Ophthalmol. 2012;57(3):202-235.

4. Meller D, Pauklin M, Thomasen H, Westkemper H, Stuehl KP. Amniotic membrane transplantation in the human eye. Dtsch Arztebl Int. 2011;108(14):243-248.

5. Doctor PP, Bhat PV, Foster CS. Subconjunctival bevacizumab for corneal neovascularization. Cornea. 2008;27(9):992-995.

6. Yakrus MA, Hernandez SM, Floyd MM, Sikes D, Butler WR, Metchock B. Comparison of methods for identification of Mycobacterium abscessus and M. chelonae isolates. J Clin Microbiol. 2001;39(11):4103-4110.
7. Odell ID, Cloud JL, Seipp M, Wittwer CT. Rapid species identification within the Mycobacterium chelonae-abscessus group by highresolution melting analysis of hsp65 PCR products. Am J Clin Pathol. 2005;123(1):96-101.

8. Ringuet $\mathrm{H}$, Akoua-Koffi $\mathrm{C}$, Honore $\mathrm{S}$, et al. hsp65 sequencing for identification of rapidly growing mycobacteria. J Clin Microbiol. 1999;37(3):852-857.

9. Scott IU, Lieb DF, Flynn HW Jr, Dessouki A, Murray TG, Miller D. Endophthalmitis caused by Mycobacterium chelonae: selection of antibiotics and outcomes of treatment. Arch Ophthalmol. 2003; 121(4):573-576.

10. Roussel TJ, Stern WH, Goodman DF, Whitcher JP. Postoperative mycobacterial endophthalmitis. Am J Ophthalmol. 1989;107(4):403-406.

11. El-Asrar AM, Tabbara KF. Chronic endophthalmitis after extracapsular cataract extraction caused by Mycobacterium chelonae subspecies abscessus. Eye (Lond). 1995;9(Pt 6):798-801.

12. Ramaswamy AA, Biswas J, Bhaskar V, Gopal L, Rajagopal R, Madhaven HN. Postoperative Mycobacterium chelonae endophthalmitis after extracapsular cataract extraction and posterior chamber intraocular lens implantation. Ophthalmology. 2000;107(7):1283-1286.

13. Gedde SJ, Scott IU, Tabandeh H, et al. Late endophthalmitis associated with glaucoma drainage implants. Ophthalmology. 2001;108(7):1323-1327.

14. Grenzebach UH, Busse H, Tötsch M, Dockhorn-Dworniczak B. Endophthalmitis induced by atypical mycobacterial infection. Ger $J$ Ophthalmol. 1996;5(4):202-206.

15. Benz MS, Murray TG, Dubovy SR, Katz RS, Eifrig CW. Endophthalmitis caused by Mycobacterium chelonae abscessus after intravitreal injection of triamcinolone. Arch Ophthalmol. 2003;121(2):271-273.

16. Ambler JS, Meisler DM, Zakov ZN, Hall GS, Spech TJ. Endogenous Mycobacterium chelonae endophthalmitis. Am J Ophthalmol. 1989;108(3):338-339.

17. Stewart MW, Alvarez S, Ginsburg WW, Shetty R, McLain WC, Sleater JP. Visual recovery following Mycobacterium chelonae endophthalmitis. Ocul Immunol Inflamm. 2006;14(3):181-183.

18. Nessar R, Cambau E, Reyrat JM, Murray A, Gicquel B. Mycobacterium abscessus: a new antibiotic nightmare. J Antimicrob Chemother. 2012;67(4):810-818.

19. Chang V, Karp CL, Yoo SH, et al. Mycobacterium abscessus endophthalmitis after Descemet's stripping with automated endothelial keratoplasty. Cornea. 2010;29(5):586-589.

20. Liesegang TJ. Herpes zoster ophthalmicus natural history, risk factors, clinical presentation, and morbidity. Ophthalmology. 2008;115 (2 Suppl):S3-S12.
Clinical Ophthalmology

\section{Publish your work in this journal}

Clinical Ophthalmology is an international, peer-reviewed journal covering all subspecialties within ophthalmology. Key topics include: Optometry; Visual science; Pharmacology and drug therapy in eye diseases; Basic Sciences; Primary and Secondary eye care; Patient Safety and Quality of Care Improvements. This journal is indexed on Submit your manuscript here: http://www.dovepress.com/clinical-ophthalmology-journal

\section{Dovepress}

PubMed Central and CAS, and is the official journal of The Society of Clinical Ophthalmology (SCO). The manuscript management system is completely online and includes a very quick and fair peer-review system, which is all easy to use. Visit http://www.dovepress.com/ testimonials.php to read real quotes from published authors. 